\title{
Significance Tests for Boosted Location and Scale Models with Linear Base-Learners
}

\author{
${ }^{1}$ Institut für medizinische Biometrie, Informatik und Epidemiologie, Medizinische Fakultät, Rheinische Friedrich-Wilhelms- \\ Universität Bonn, Bonn, Germany, E-mail: tobias.hepp@uk-erlangen.de \\ ${ }^{2}$ Institut für Medizininformatik, Biometrie und Epidemiologie, Medizinische Fakultät, Friedrich-Alexander-Universität \\ Erlangen-Nürnberg, Erlangen, Cermany, E-mail: tobias.hepp@uk-erlangen.de
}

\begin{abstract}
:
Generalized additive models for location scale and shape (GAMLSS) offer very flexible solutions to a wide range of statistical analysis problems, but can be challenging in terms of proper model specification. This complex task can be simplified using regularization techniques such as gradient boosting algorithms, but the estimates derived from such models are shrunken towards zero and it is consequently not straightforward to calculate proper confidence intervals or test statistics. In this article, we propose two strategies to obtain $p$-values for linear effect estimates for Gaussian location and scale models based on permutation tests and a parametric bootstrap approach. These procedures can provide a solution for one of the remaining problems in the application of gradient boosting algorithms for distributional regression in biostatistical data analyses. Results from extensive simulations indicate that in low-dimensional data both suggested approaches are able to hold the type-I error threshold and provide reasonable test power comparable to the Wald-type test for maximum likelihood inference. In high-dimensional data, when gradient boosting is the only feasible inference for this model class, the power decreases but the type-I error is still under control. In addition, we demonstrate the application of both tests in an epidemiological study to analyse the impact of physical exercise on both average and the stability of the lung function of elderly people in Germany.
\end{abstract}

Keywords: boosting, GAMLSS, regularized regression, permutation test, parametric bootstrap

DOI: $10.1515 / \mathrm{ijb}-2018-0110$

Received: October 25, 2018; Revised: January 31, 2019; Accepted: March 21, 2019

\section{Introduction}

Regression models play a pivotal role in analyzing the relationship between an outcome of interest and multiple explanatory variables in many research areas. While the distribution of the response vector $\boldsymbol{y}$ can usually be described by multiple parameters, the main focus of most regression problems lies on using a set of input variables $X$ to model only the mean. Although there exist very flexible tools like generalized additive models (GAM) [1] to model the pattern of this dependency, focusing solely on the expected value of a distribution may lead to overlooking patterns in the data that are for instance responsible for differences in the residual variance of Gaussian regression or the dispersion parameters for negative binomial regression. For this purpose, generalized additive models for location, scale and shape (GAMLSS) [2] extend the classical GAM by defining additional additive predictors to model the dependency on the covariates of up to $k=1, \ldots, K$ distribution parameters $\theta_{k=1, \ldots, K}$ :

$$
g_{k}\left(\boldsymbol{\theta}_{k}\right)=\beta_{0 \theta_{k}}+\sum_{j=1}^{p_{k}} f_{j \theta_{k}}\left(\boldsymbol{x}_{k j}\right)=\eta_{\theta_{k}}
$$

Here, $g_{k}(\cdot)$ denotes known monotonic link functions and $\boldsymbol{x}_{k 1}, \ldots, \boldsymbol{x}_{k p_{k}}$ the $p_{k}$ (possibly different) input variables for each predictor $\boldsymbol{\eta}_{\theta_{k}}$ and $f_{j \theta_{k}}(\cdot)$ describes the shape of the effect of $\boldsymbol{x}_{k j}$ on $\boldsymbol{\theta}_{k}$. Set up properly, this model class therefore not only serves to explicitly address violated model assumptions such as heteroskedasticity, but offers additional insights on the dependence structure between input variables and the outcome beyond the expectation of its distribution.

Since GAMLSS's are originally fitted via penalized maximum likelihood algorithms [3], proper specification of all additive predictors quickly becomes a challenging task in practice. Therefore, Mayr et al. [4] proposed an 
alternative approach to GAMLSS inference based on a multivariate extension of gradient boosting [5]. The concept of boosting has been originally developed in the area of machine learning and was later adapted to the framework of common statistical regression models [6-9] and continuously developed further [10-13]. By using the iterative structure of boosting algorithms as regularizing constraints on the model to be estimated, this approach thereby implicitly allows for variable selection and to fit even over-parameterized models to highdimensional datasets with $p \gg n$.

While gradient boosting provides a very flexible and versatile approach to model-based inference, it shares the fate of many other regularization techniques in that there is no straightforward way to compute proper test-statistics based on distributional assumptions for single regression coefficients like the t-test. This is due to the bias induced by shrinkage, which also affects otherwise viable alternatives such as bootstrap confidence intervals [13]. Although tuning the model results in a set of variables considered 'informative' based on whether they have been selected at least once by the algorithm or not at all, using for example cross-validation usually leads to the inclusion of many false positives, i.e. variables without true effect on the outcome [14-16].

To overcome this limitation, Mayr et al. [17] proposed the application of permutation tests to analyse the effect of different medical devices on accuracy and random measurement error. The idea of using randomization techniques to test the effect estimates in regression models has been suggested very early [18] and considered especially useful in the presence of small sample size or disputable asymptotic assumptions in general. While different strategies with respect to the choice of the object to be permutated and the considered test statistic exist (see Kennedy and Cade [19] for an overview), Mayr et al. focused on permuting a single regressor variable $x_{j}$ which is then used instead of its original to fit the model on the data. Since the variable is known to be uncorrelated with the response after random permutation, repeating this procedure multiple times generates a distribution of the corresponding effect estimate under the null-hypothesis $H_{0}: \beta_{j}=0$, i.e. $\boldsymbol{x}_{j}$ has no true effect on the response.

Although the application scenario already involves the use of location and scale models from the Gaussian distribution as in the GAMLSS framework, this approach only works because the choice of the measurement device in an experimental setting is independent of other covariates, but will fail to hold the type-I error-bound in correlated designs since using the 'blank' effect estimate as test statistic ignores the loss of reliability in single estimates resulting from multicollinearity.

As a consequence, we propose two alternative strategies to obtain $p$-values for boosted location and scale models in the presence of correlated covariates. The first, the residual permutation test, is a slightly altered version of the 'simple' permutation test described above. By permuting the residuals of a linear regression of the variable of interest on all available covariates instead of the variable itself, independence to the covariates is ensured. The second uses the parametric bootstrap to create multiple new samples by ignoring the estimated impact of the variable of interest on a distribution parameter of the response.

Both procedures are described in more detail after a brief introduction to boosted GAMLSS [4] in Section 2. Section 3 provides the results of comprehensive simulations to investigate both the type-I-error rate and power of the suggested tests with respect to varying settings regarding the effect strength on expectation and variance. Finally, both tests are used with models applied on survey data to evaluate the contribution of physical training to both expectation and variance of lung function in middle-aged and elderly people in Germany in Section 4 .

\section{Methods}

\subsection{Boosting GAMLSS}

The main idea behind all boosting algorithms is to repeatedly fit weak prediction functions to the data while each time emphasizing those parts of the response that were insufficiently predicted in previous iterations to eventually combine them into a strong ensemble [20]. Following a model-based boosting approach [8], these base-learners are usually regression-type functions of a single input variable $\boldsymbol{x}_{j}$ (e.g. simple linear effects or splines), which are fit to the negative gradient of a specified loss-function (e.g. residual sum of squares or the negative log-likelihood). Then, starting from an empty model, the algorithm updates only the best performing base-learner in each iteration, successively expanding the model in the direction where the loss is reduced most. This general concept allows to combine any base-learner with any suitable loss function, leading to a large number of different model classes (e.g., quantile regression [21], conditional transformation models [22] or geoadditive regression [23]) that can be fitted by boosting.

Algorithm 1 gamboostLSS (Mayr et al. [4])

Initialize 
- Set the iteration counter $m=0$ and initialize all additive predictors $\hat{\boldsymbol{\eta}}_{\theta_{k}}^{[0]}$ with offset values, e.g. $\hat{\eta}_{\theta_{k} i}^{[0]}=\arg \max _{c} \sum_{i=1}^{n} l\left(y_{i}, \eta_{\theta_{k} i}=c\right)$ for $k=1, \ldots, K$ and $i=1, \ldots, n$

- Specify a set of base-learners $h_{1 \theta_{k}}(\cdot), \ldots, h_{p_{k} \theta_{k}}(\cdot)$ for each distribution parameter $\boldsymbol{\theta}_{k}$, where $p_{k}$ is the cardinality of the set of base-learners specified for $\boldsymbol{\theta}_{k}$

Boosting in multiple dimensions

1. While $m<m_{\text {stop }_{k}}$ for all $k$, set $m:=m+1$ and $k:=0$

2. While $k<K$, set $k:=k+1$

(a) If $m \leq m_{\text {stop }_{k}}$

i. Compute the negative partial derivative:

$$
\boldsymbol{u}_{k}^{[m-1]}=-\left.\frac{\partial \rho(\boldsymbol{y}, H)}{\partial \boldsymbol{\eta}_{\theta_{k}}}\right|_{H=\left[\hat{\boldsymbol{\eta}}_{\theta_{1}}^{[m-1]} \ldots \hat{\boldsymbol{\eta}}_{\theta_{K}}^{[m-1]}\right]}
$$

ii. Fit all base learners specified for $\eta_{\theta_{k}}$ separately to the negative gradient vector

$$
\left(\boldsymbol{u}_{k}^{[m-1]}, \boldsymbol{x}_{k j}\right) \stackrel{\text { base-leaner }}{\longrightarrow} \hat{h}_{j \theta_{k}}(\cdot)
$$

and find the one with the best fit:

$$
j^{*}=\underset{1 \leq j \leq p_{k}}{\arg \min } \sum_{i=1}^{n}\left(u_{i k}^{[m-1]}-\hat{h}_{j \theta_{k}}(\cdot)\right)^{2}
$$

iii. Update $\hat{\boldsymbol{\eta}}_{\theta_{k}}^{[m-1]}$ by a small step-length $0<\mathrm{sl} \ll 1$ :

$$
\hat{\boldsymbol{\eta}}_{\theta_{k}}^{[m-1]}:=\hat{\boldsymbol{\eta}}_{\theta_{k}}^{[m-1]}+\mathrm{sl} \cdot \hat{h}_{j \theta_{k}}(\cdot),
$$

(b) Set $\hat{\boldsymbol{\eta}}_{\theta_{k}}^{[m]}:=\hat{\boldsymbol{\eta}}_{\theta_{k}}^{[m-1]}$

Applied to the GAMLSS framework, this approach results in the iterative steps presented in Algorithm 1, which is implemented in the R add-on package gamboost LSS [24, 25]. For the sake of generalizability, $h_{j \theta_{k}}(\cdot)$ denotes all types of implemented base-learners for the different distribution parameters and hence adopts the role of $f_{j \theta_{k}}(\cdot)$ from the introduction. For the remainder of this article, however, we only use the same linear prediction functions for all distribution parameters, so that $\boldsymbol{x}_{k j}=\boldsymbol{x}_{j}$ for all $k$ and $h_{j \theta_{k}}\left(\boldsymbol{x}_{j}\right)=\beta_{j \theta_{k}} \boldsymbol{x}_{j}$.

After initialization, all predictors are iteratively updated by selecting the base-learner with the best fit on the negative gradient of the negative log-likelihood with respect to the order of $\theta_{k}$. In the case of a Gaussian response with $\theta_{1 i}=\mu_{i}$ and $\theta_{2 i}=\sigma_{i}$, this means each iteration of the algorithm starts with updating the predictor for the expectation $\hat{\eta}_{\mu i}$ first before updating the variance component via $\hat{\eta}_{\sigma i}$. By stopping the algorithm before convergence, the model is effectively regularized and consequently shows similar behaviour as well as results as the probably more common lasso, including biased effect estimates [26, 27]. Hence, proper tuning of the algorithm allows for variable selection even in high-dimensional datasets where the number of candidate variables $p$ exceeds the number of observation $n$, i.e. $p \gg n$.

Since using the same $m_{\text {stop }}$ for all distribution parameters does not necessarily lead to the best solution with respect to loss reduction, separate stopping points can be determined in order to find the best model with the desired properties. These differing $m_{\text {stop }_{k}}$ are usually determined using cross-validation or related approaches over a $K$-dimensional grid of potential stopping values. However, this often leads to the inclusion of many false positive variables in the cross-validated solution due to the greedy nature of the algorithm and the explicit focus on minimizing the prediction error on the folds not used for estimating the model [28]. Therefore, boosted GAMLSS greatly benefit from alternative approaches able to evaluate the likelihood of an effect being truly informative for the parameter of an response distribution and being applicable in all kinds of data settings.

\subsection{Residual permutation test}

A potential approach to test the significance of regularized effect estimates of model-based boosting algorithms in the presence of correlated covariates is to slightly alter the proposed procedure for the permutation test [17] by replacing $x_{j}$ with the residuals $\hat{\boldsymbol{\epsilon}}$ of the ordinary least squares solution of $\boldsymbol{x}_{j}$ given all other candidate variables 
$\boldsymbol{x}_{m}$ for all $m \neq j[29,30]$. Since the residuals are by design uncorrelated to all other variables except for the outcome $\boldsymbol{y}$, the conditions necessary for the approach to work are restored. Then, repeatedly permuting $\hat{\boldsymbol{\epsilon}}$ and refitting the model generates an empirical distribution of $\hat{\beta}_{\hat{\epsilon}}$ under the null-hypothesis, which can subsequently be used to estimate the $p$-value by comparing it to the model fit with the original residuals. This means that in order to conclude that there is a significant effect of the examined $\boldsymbol{x}_{j}$ on the response variable for $\alpha=0.05$, the absolute value of the effect estimate of its residuals should be larger than at least $95 \%$ of the absolute estimates derived from their randomly permuted versions. Algorithm 2 transfers the general concept to the estimation of $p$-values for boosted GAMLSS. Since using gradient boosting for inference involves shrinkage of all effect estimates, it should be noted that the degree of regularization is to be held constant for all estimated coefficients. Therefore, the same stopping iteration $m_{\text {stop }}$ has to be used for all model fits in order to provide a fair comparison [31].

In addition to substituting $\hat{\boldsymbol{\epsilon}}$ for $\boldsymbol{x}_{j}$ if covariates are correlated, there is another important aspect to be considered if the same variable $\boldsymbol{x}_{j}$ is used to model multiple distribution parameters. While it may at first seem efficient to use the permuted residuals in all predictors $\hat{\boldsymbol{\eta}}_{\theta_{k}}$ in order to compute the $p$-values for all $\hat{\beta}_{\hat{\epsilon} \theta_{k}}$ simultaneously, we recommend to permute $\hat{\epsilon}$ only for a single predictor and use the original residuals to model the other distribution parameters instead. The reason for this is that in the case of a Gaussian distributed variable, for example, the estimation of $\hat{\beta}_{j \sigma}$ clearly depends on the current value of $\hat{\beta}_{j \mu}$. Hence, using the permutations in $\hat{\eta}_{\mu}$ is more or less equivalent to completely removing the term, thereby directly influencing the estimation of $\hat{\beta}_{j \sigma}$.

While this modified permutation test solves the problem associated with correlated predictor variables, it should be noted that computing the residuals by means of ordinary least squares is not possible if $p>n$. As a consequence, using the residual permutation test is restricted to low-dimensional analyses even for boosting algorithms, thereby limiting the scope of potential applications. Since the residuals are only computed for a single variable and the coefficient itself is used as the test statistic, it is furthermore not applicable to test nonlinear effects or multiple hypotheses at once.

\subsection{Parametric bootstrap}

An alternative approach to hypothesis testing may be achieved via the bootstrap. As for permutation tests, the basic idea of bootstrapping experienced several developments over the past decades [32,33]. The probably most common bootstrap is the creation of multiple new samples by repeatedly drawing $n$ observations from the original data with replacement. Then, computing the desired statistic separately on the new data sets produces an empirical bootstrap distribution from which a bootstrap confidence interval can be derived. For a short discussion within the context of boosting algorithms see Hofner et al. [13], for likelihood-based inference on gamlss see Stasinopoulos et al. [34].

Algorithm 2 Residual permutation test for boosted Gaussian location and scale models

1. Compute the ordinary least squares solution for

$$
\boldsymbol{x}_{k j}=\gamma_{0}+\sum_{m \neq j} \gamma_{m} \boldsymbol{x}_{k m}+\hat{\boldsymbol{\epsilon}}
$$

2. Substitute $\hat{\epsilon}$ for $\boldsymbol{x}_{k j}$ in $\boldsymbol{\eta}_{\theta_{k}}$

3. Fit the model to the data via boosting and store $\hat{\beta}_{\hat{\epsilon} \theta_{k}}$

4. Repeat $B$ times:

(a) Shuffle $\hat{\epsilon}$ and refit the model using the same $m_{\text {stop }}$ as in Step 2

(b) Store the estimated coefficients $\hat{\beta}_{\hat{\epsilon} \theta_{k}}^{(b)}$

5. Compare the estimates with the ones from the original model:

$$
p-\operatorname{value}\left(\hat{\beta}_{j \theta_{k}}\right)=\frac{1}{B} \sum_{b=1}^{B} \mathrm{I}\left(\left|\hat{\beta}_{\hat{\epsilon} \theta_{k}}^{(b)}\right| \geq\left|\hat{\beta}_{\hat{\epsilon} \theta_{k}}\right|\right)
$$

Our approach to generate significance tests focuses on the parametric bootstrap (see [35] in the context of random effect estimates). Instead of creating new data sets by sampling from the full rows in the original data, the parametric bootstrap generates a new response variable zby drawing from a distribution under the null-hypothesis. This is accomplished by fitting a restricted model in addition to the full model to the data which neglects $\boldsymbol{x}_{j}$ in 
the estimation of $\hat{\eta}_{\theta_{k}}$. In the case of Gaussian location and scale models, this leads to the new response vector $z$ being drawn from

$$
z_{i} \sim \mathcal{N}\left(\hat{\eta}_{\mu i}^{[\text {null }]}, \exp \left(\hat{\eta}_{\sigma i}^{[\text {null }] 2}\right)\right)
$$

where the superscript indicates that the predictors originate from the constrained null-model. Subsequently, both full and constrained model are then fitted to $\boldsymbol{z}$ and the likelihood ratio statistic is computed. Since $\boldsymbol{x}_{j}$ plays no part in the estimation of $\hat{\eta}_{\theta_{k}}^{\text {[null] }}$, any advantage of the full model in comparison to the constrained model can therefore be attributed to chance alone. Repeating this process several times hence provides a distribution of the likelihood ratio statistic under the null hypothesis $H_{0}: \beta_{j \theta_{k}}=0$. Afterwards, the $p$-value of the estimate of interest can be derived from the proportion of likelihood ratio statistics computed on the simulated data exceeding that from the models fitted to the original data.

An overview of all steps in the context of boosted Gaussian location and scale models is provided in Algorithm 3. Similar to the permutation tests described in the previous subsection, the same stopping iteration $m_{\text {stop }}$ should be used for all models to allow for a fair comparison except for the null-model used to create the new responses $z_{i}$, which has to be fitted until convergence in order to catch all potential contributions to the prediction provided by the complete set of candidate variables.

Making use of the likelihood ratio as test statistic further allows the parametric bootstrap to be used to test also non-linear effects or multiple hypotheses at once. In contrast to the residual permuation approach, the parametric bootstrap is further also applicable when analyzing high-dimensional data. However, some caution is advised in these settings, as this complicates the estimation of the null-model used to draw the new bootstrap samples. Therefore, the true underlying regression function should at least be sparse in terms of the $L_{1}$-norm of the regression coefficients to ensure consistent estimations [36].

\footnotetext{
Algorithm 3 Parametric bootstrap for boosted Gaussian location and scale models

1. Fit the full model via boosting to the original response vector $y$

2. Fit the constrained null-model to $\boldsymbol{y}$, leaving out $\boldsymbol{x}_{j}$ for only one $\hat{\boldsymbol{\eta}}_{\theta_{k}}$ but using the same $m_{\text {stop }}$ as in the full model

3. Compute the likelihood ratio $L R$ of the resulting models

4. Fit the null-model until convergence to estimate $\hat{\eta}_{\mu i}^{[\text {[null] }}$ and $\hat{\eta}_{\sigma i}^{[\text {null] }}$
}

5. Repeat $B$ times:

(a) Draw $n$ new observations $z_{i} \sim \mathcal{N}\left(\hat{\eta}_{\mu i}^{\text {[null] }}, \exp \left(\hat{\eta}_{\sigma i}^{[\text {null]2 }}\right)\right)$

(b) Fit both full and constrained model to the new response vector $\boldsymbol{z}$ using the same $m_{\text {stop }}$ as in Step 1

(c) Compute and store the likelihood ratio $L R^{(b)}$

6. Compare the likelihood ratios:

$$
\hat{p} \text {-value }\left(\hat{\beta}_{j \theta_{k}}\right)=\frac{1}{B} \sum_{b=1}^{B} \mathrm{I}\left(L R^{(b)} \geq L R\right)
$$

\section{Simulation study}

\subsection{Ceneral setting}

To investigate whether or not the suggested tests work as intended, we examined the performance of both methods using simulated data and compared them to the Wald-type test implemented in the gamlss-package [3] as well as the $\chi^{2}$-test based on the likelihood ratios. As a consequence, the general setting is required to be low-dimensional for both penalized maximum likelihood inference and residual permutation test to work. To be precise, all simulated core data sets $X$ are composed of $p=5$ predictor variables for $n=200$ cases. The response variable $y$ is then sampled from $\mathcal{N}(\boldsymbol{\mu}, \boldsymbol{\sigma})$ with linear prediction functions for both expectation and variance as follows:

$$
\boldsymbol{\mu}=2+\sum_{j=1}^{p} \beta_{j \mu} \boldsymbol{x}_{j} \quad \text { and } \quad \boldsymbol{\sigma}=\exp \left(\sum_{j=1}^{p} \beta_{j \sigma} \boldsymbol{x}_{j}\right)
$$

Due to using the log-link to ensure positivity, the variance term is susceptible to outliers. For this reason, all predictor variables are generated to follow a standard uniform distribution $\mathcal{U} \sim(0,1)$ by using the cumulative 
density function of the Gaussian distribution $\Phi()$ so that

$$
X=\left[\Phi\left(z_{1}\right) \Phi\left(z_{2}\right) \ldots \Phi\left(z_{p}\right)\right],
$$

where the auxiliary matrix $Z=\left[z_{1} z_{2} \ldots z_{p}\right]$ is drawn from a multivariate normal distribution $\mathcal{N}(\mathbf{0}, \Sigma)$. Setting $\Sigma_{i j}=2 \sin \left((2 / 3)^{|i-j|} \frac{\pi}{6}\right)$ then leads to all data sets $X$ being quasi multivariate uniformly distributed with expected Toeplitz correlation structure $\rho_{\boldsymbol{x}_{i} \boldsymbol{x}_{j}}=(2 / 3)^{|i-j|}$ for all $1 \leq i, j \leq p[37,38]$.

Since one of the advantages of model-based boosting algorithms is their applicability in high-dimensional scenarios, we also expanded the general setting by adding 300 uninformative uniformly distributed variables to the design. These expanded settings thus consist of $p=305$ predictor variables with $n=200$ cases. Moreover, we further simulated data in which the interesting effect is actually non-linear. However, the results are absolutely consistent with the results from the strictly linear cases, so we will focus on the latter in the following chapters and provide our additional results in the supplements (Figure S3 and Figure S4).

\subsection{Type-I-error}

First, we focused on investigating the type-I-error rate, i.e. the probability of erroneously rejecting $H_{0}$ by assuming a significant effect that does in fact not exist. Therefore, we started by first considering multiple scenarios regarding the true values of $\beta_{1 \mu}$ and $\beta_{1 \sigma}$ to reflect these situations, while fixing $\beta_{2 \mu}=-1$ and $\beta_{2 \sigma}=0.5$ to add a correlated informative covariate to all designs. Finally, $\boldsymbol{x}_{3}, \boldsymbol{x}_{4}$ and $\boldsymbol{x}_{5}$ are noise variables that influence neither expectation nor variance of the response $\boldsymbol{y}$ but are of course still correlated to the other covariates in $X$.

We then differentiated between the following three scenarios:

Setting 00: $\beta_{1 \mu}=0$ and $\beta_{1 \sigma}=0$ ( $\boldsymbol{x}_{1}$ completely uninformative)

Setting 10: $\beta_{1 \mu}=1$ and $\beta_{1 \sigma}=0$ ( $\boldsymbol{x}_{1}$ influences only the expected value)

Setting 01: $\beta_{1 \mu}=0$ and $\beta_{1 \sigma}=1$ ( $\boldsymbol{x}_{1}$ influences only the variance)

For each setting, 1000 simulation runs were executed with the number of permutations or bootstrap samples both set to $B=1000$. Furthermore, an important aspect to be considered is the potential impact of shrinkage on the performance of both suggested approaches due to early stopping of the boosting algorithms. For this reason, we additionally examined the performance with regard to stopping at iterations $m_{\text {stop }} \in\{25,50,100,200\}$. However, it should be noted that higher values for $m_{\text {stop }}$ may be necessary in many practical scenarios, since all possibly informative variables have at least to be given the chance of being selected at all.

Table 1 contains the estimated type-I-error rate for all simulated settings by reporting the proportion of $p$ values smaller or equal to the commonly used significance threshold $\alpha=0.05$. As anticipated by Mayr et al. [17], the 'naive' permutation approach fails in all three simulation settings by rejecting the true $H_{0}$ in clearly more than the admitted $5 \%$ of all simulations for both $\beta_{1 \mu}$ and $\beta_{1 \sigma}$ due to the presence of correlated covariates. In contrast, substituting the residuals for $\boldsymbol{x}_{1}$ before applying the permutation test delivers results close to the desired proportion, in some settings even outperforming the Wald-type test implemented in gamlss. The same applies to the parametric bootstrap, which delivers proportions even closer to the desired $5 \%$. Since the performance of the $\chi^{2}$-test is almost exactly on par with the Wald-type test, we only report the latter but provide all results in the supplements (Figure 4).

Table 1: Estimated type-I-error rate regarding the true effect of $x_{1}$ on either $\mu$ or $\sigma$ for all simulation settings. Results of penalized ML do not depend on $m$ and are therefore only mentioned once. When $p>n$, only simple permutation and the parametric bootstrap can be applied, other cells remain empty.

\begin{tabular}{|c|c|c|c|c|c|c|c|c|c|c|}
\hline \multirow[t]{2}{*}{$\mathrm{p}$} & \multirow[t]{2}{*}{$m$} & \multirow[t]{2}{*}{ Setting } & \multicolumn{2}{|c|}{ Simple Perm. } & \multicolumn{2}{|c|}{ Residual Perm. } & \multicolumn{2}{|c|}{ Param. Boots. } & \multicolumn{2}{|c|}{ Penalized ML } \\
\hline & & & $\beta_{1 \mu}$ & $\beta_{1 \sigma}$ & $\beta_{1 \mu}$ & $\beta_{1 \sigma}$ & $\beta_{1 \mu}$ & $\beta_{1 \sigma}$ & $\beta_{1 \mu}$ & $\beta_{1 \sigma}$ \\
\hline \multirow[t]{9}{*}{5} & \multirow{3}{*}{25} & 00 & 0.150 & 0.105 & 0.059 & 0.051 & 0.059 & 0.058 & 0.070 & 0.057 \\
\hline & & 10 & . & 0.095 & . & 0.051 & . & 0.060 & . & 0.057 \\
\hline & & 01 & 0.197 & . & 0.064 & . & 0.052 & . & 0.062 & 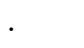 \\
\hline & \multirow{3}{*}{50} & 00 & 0.110 & 0.095 & 0.060 & 0.048 & 0.057 & 0.057 & & \\
\hline & & 10 & . & 0.100 & . & 0.056 & . & 0.056 & & \\
\hline & & 01 & 0.187 & 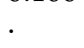 & 0.079 & . & 0.046 & . & & \\
\hline & \multirow{3}{*}{100} & 00 & 0.098 & 0.125 & 0.055 & 0.053 & 0.056 & 0.057 & & \\
\hline & & 10 & . & 0.122 & . & 0.052 & . & 0.054 & & \\
\hline & & 01 & 0.126 & . & 0.078 & . & 0.051 & . & & \\
\hline
\end{tabular}




\begin{tabular}{|c|c|c|c|c|c|c|c|c|}
\hline & 200 & $\begin{array}{l}00 \\
10 \\
01\end{array}$ & $\begin{array}{l}0.112 \\
\cdot \\
0.098\end{array}$ & $\begin{array}{l}0.150 \\
0.158 \\
.\end{array}$ & $\begin{array}{l}0.058 \\
\cdot \\
0.077\end{array}$ & $\begin{array}{l}0.057 \\
0.054 \\
\text {. }\end{array}$ & $\begin{array}{l}0.056 \\
\cdot \\
0.051\end{array}$ & $\begin{array}{l}0.050 \\
0.053 \\
.\end{array}$ \\
\hline \multirow[t]{12}{*}{305} & \multirow{3}{*}{25} & 00 & 0.136 & 0.139 & & & 0.066 & 0.042 \\
\hline & & 10 & . & 0.149 & & & $\cdot$ & 0.043 \\
\hline & & 01 & 0.127 & . & & & 0.056 & . \\
\hline & \multirow{3}{*}{50} & 00 & 0.171 & 0.157 & & & 0.064 & 0.040 \\
\hline & & 10 & . & 0.162 & & & . & 0.043 \\
\hline & & 01 & 0.163 & . & & & 0.053 & . \\
\hline & \multirow{3}{*}{100} & 00 & 0.160 & 0.147 & & & 0.038 & 0.041 \\
\hline & & 10 & . & 0.157 & & & . & 0.037 \\
\hline & & 01 & 0.204 & . & & & 0.038 & . \\
\hline & \multirow{3}{*}{200} & 00 & 0.126 & 0.137 & & & 0.001 & 0.023 \\
\hline & & 10 & . & 0.147 & & & . & 0.012 \\
\hline & & 01 & 0.203 & . & & & 0.017 & . \\
\hline
\end{tabular}

Except for slightly declining proportions in setting 01 for the residual permutation test, there is no evidence for a systematic impact of the stopping iteration on the performance regarding the type-I-error rate. This is somewhat different regarding the high-dimensional settings. Here, the parametric bootstrap is the only promising solution and shows in fact quite reasonable results. However, it seems that the proportion of $p$-values below or equal to 0.05 declines when increasing the number of iterations in the boosted models, which becomes evident for $m_{\text {stop }}=200$. Although rejection rates below $\alpha$ are not problematic for the individual test decisions from a practical viewpoint, this behaviour deserves further investigation.

\subsection{Statistical power}

The second important feature of statistical hypothesis tests is their power, i.e. the probability to correctly identify a truly existing effect by producing a $p$-value below or equal to the predefined $\alpha$-threshold. Therefore, we repeated the simulation study, each time slightly changing the true values $\beta_{1 \mu}$ or $\beta_{1 \sigma}$ in steps of 0.2 while holding the other one constant. Since this involves considerable computational demands, we still executed 1000 simulation runs for each configuration but lowered $B$ to 200 for both residual permutation and parametric bootstrap.

Starting with the low dimensional setting, Figure 1 illustrates the performance of both tests compared to the Wald-type-test implemented in the gamlss-package, where all plots on the left side result from changing the true effect on the expectation of the response and the plots on the right side from changing the true effect on its variance. Moreover, the first row for each test represents the scenarios where $x_{1}$ has no impact on the other parameter of the distribution of $\boldsymbol{y}$ while the second row shows more difficult settings with $\beta_{1 \sigma}$ fixed at 1 for the evaluation of $\beta_{1 \mu}$ and $\beta_{1 \mu}$ fixed at 5 for the evaluation of $\beta_{1 \sigma}$. The performance of the $\chi^{2}$-test still closely matches that of the Wald-type test and is reported in the supplements (Figure S1). 

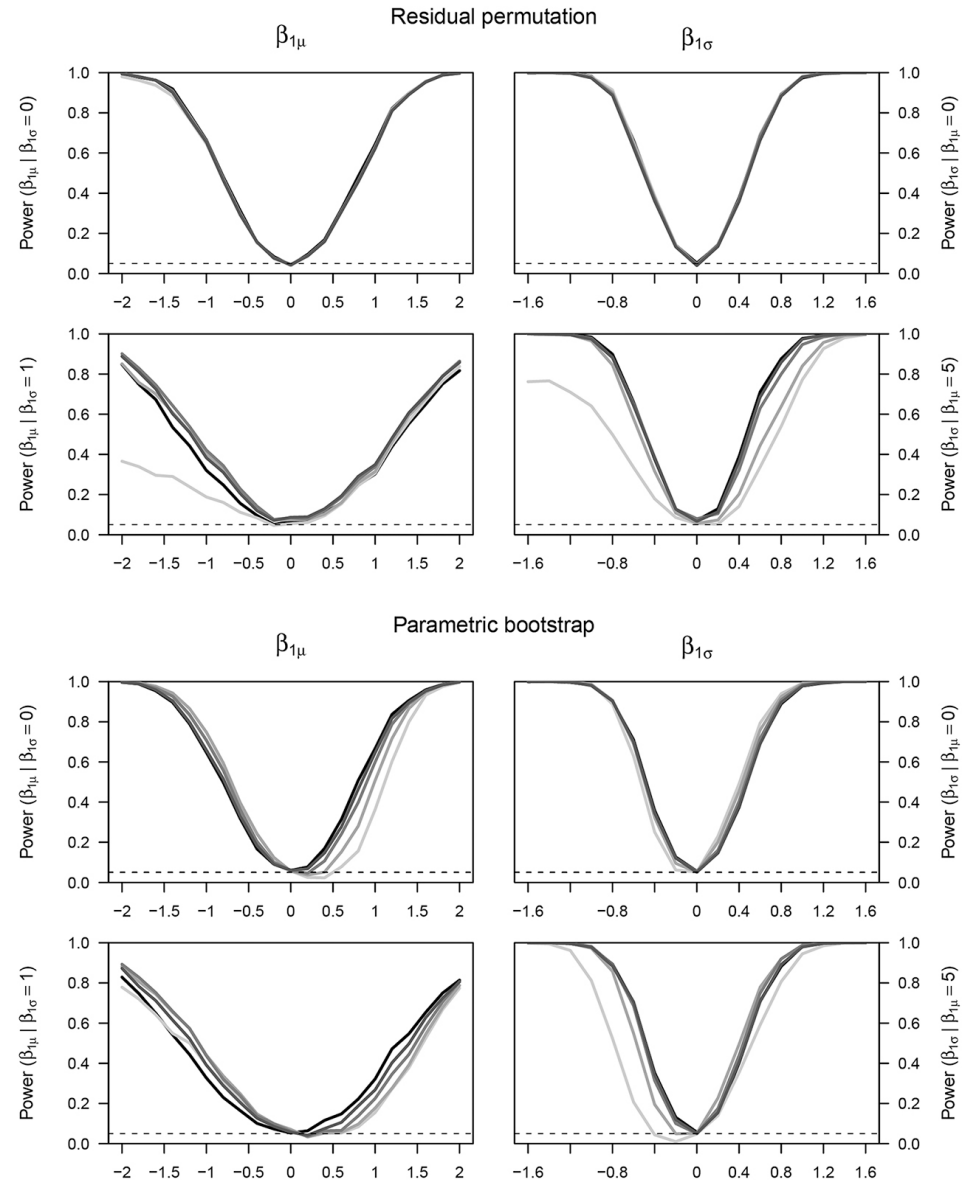

Figure 1: Test power for the residual permutation test and parametric bootstrap in the low-dimensional setting estimated via simulations. Lighter shades of grey represent a higher degree of regularization, while the black line shows the results of the gamlss-package. Plots on the left side evaluate the power with respect to the effect size of $\beta_{1 \mu}$ with $\beta_{1 \sigma}$ set to either 0 or 1 , whereas the plots on the right side evaluate a range for $\beta_{1 \sigma}$ in scenarios with $\beta_{1 \mu}$ constant at 0 or 5 .

From a general perspective, it can be seen that the smaller the absolute true value becomes, the less likely it is to be identified as truly significant influence on $\mu$ or $\sigma$. As suggested by the previous section, this culminates in the type-I-error rate of approximately $5 \%$ represented by the fine dashed line when reaching $\beta_{1 \mu}=0$ and $\beta_{1 \sigma}=0$, irrespective of both the effect on the other parameter and the number of iterations. Starting with the results for the residual permutation tests, it seems that the degree of regularization has no systematic effect on the power in the more simple setting, but the performance suffers if the algorithm is stopped very early if $x_{1}$ is also informative for the other distribution parameter. This applies in particular to the tests regarding $\beta_{1 \sigma}$, as the identification of the effect depends at least to some degree on the values of $\beta_{1 \mu}$ and is therefore more difficult if this parameter is still subject to strong shrinkage. As a consequence, we again emphasize the need to run the algorithm long enough before computing the $p$-values, as the simulations further show that the estimated test power is almost exactly on top of or at least very close to the results of the gamlss- "gold-standard" if the model is not too strictly regularized. Most interestingly, however, while setting $\beta_{1 \sigma}=1$ increases the overall variance and consequently leads to a broader 'valley' of test power for $\beta_{1 \mu}$, the residual permutation test with $m_{\text {stop }} \geq 50$ even outperforms the Wald-type-test in this scenario.

While these findings are for the main part also true for the results of the parametric bootstrap, there seems to be a shift in test power that intensifies for lower values of $m_{\text {stop }}$. This asymmetry in power superiority becomes particularly apparent by looking at the differences between the proportions resulting from the bootstrap and those of the gamlss-package (Figure S2). Moreover, the direction of this shift differs for location and scale: While small positive values of $\beta_{1 \mu}$ lead to comparably lower test power with strong regularization, the opposite holds for $\beta_{1 \sigma}$.

This phenomenon arises as a result of the parametric bootstrap approaching the correlation problem from a different angle, as - in contrast to the residual permutation test - creating new response vectors focuses on eliminating the correlation between negative gradient and the predictor variable of interest instead of that between the covariates. Depending mainly on the signs but to some degree also on the strength of the correlation between covariates and the negative gradient, this results in either over-emphasizing or attenuating the dif- 
ference in the log-likelihoods in early iterations. Reminding $\beta_{2 \mu}=-1$ and $\beta_{2 \sigma}=0.5$, the last row of Figure 1 clearly illustrates this pattern.

The asymmetric power regarding whether the true effect size is positive or negative is also observable in the expanded high-dimensional scenarios (Figure 2). Not surprisingly, the overall power of the test is smaller due to random correlation occuring between $x_{1}$ and the large number of additional variables in the set. More interestingly, however, is the fact that larger values of $m_{\text {stop }}$ seem to additionaly reduce the test power and for the variance parameter the test even seems to lose all power if the true $\beta_{1 \sigma}$ drifts too far into the negative. A potential explanation for the latter is that considering the general simulation setting, very small values of $\beta_{1 \sigma}$ result in very small variances in some regions of the response variable. Then, in case of high-dimensional data, large values of $m_{\text {stop }}$ force the algorithm to drastically overfit and increase the likelihood based on spurious correlations. If $\beta_{1 \sigma}$ is positive the variance in $y$ will be higher overall, thereby attenuating this phenomenon and explaining the asymmetry in Figure 2.

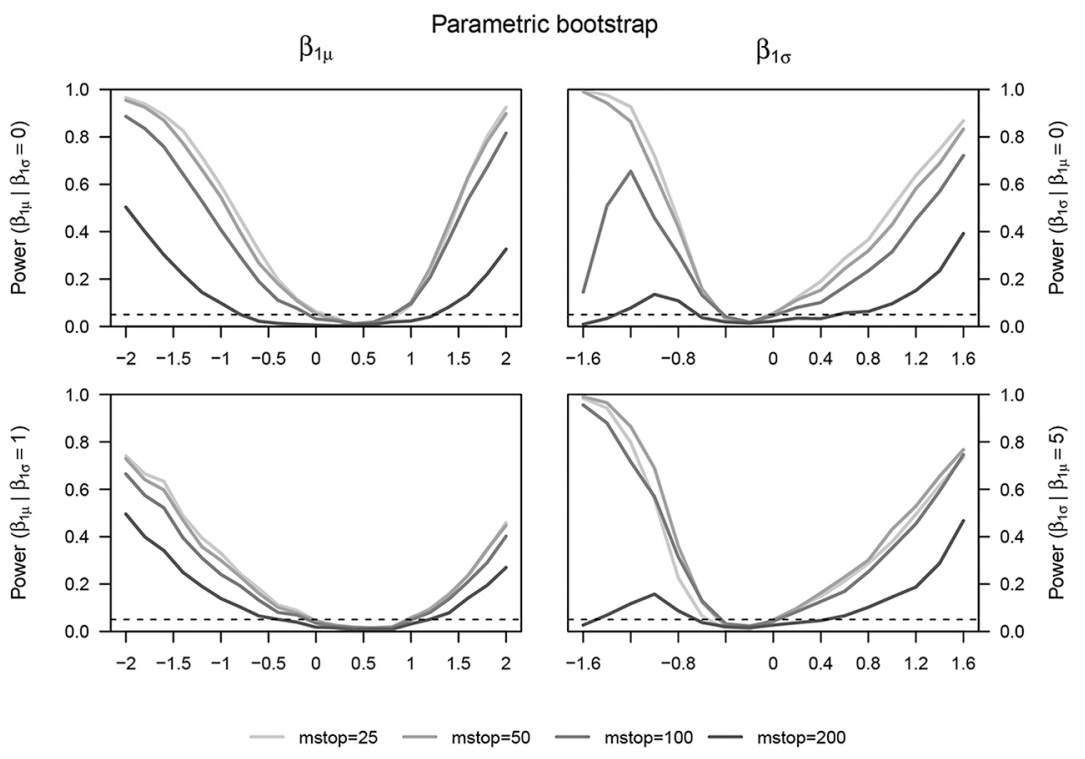

Figure 2: Test power for the parametric bootstrap in the high-dimensional setting estimated via simulations. Lighter shades of grey represent a higher degree of regularization. Plots on the left side evaluate the power with respect to the effect size of $\beta_{1 \mu}$ with $\beta_{1 \sigma}$ set to either 0 or 1 , whereas the plots on the right side evaluate a range for $\beta_{1 \sigma}$ in scenarios with $\beta_{1 \mu}$ constant at 0 or 5 .

Irrespective of the apparent influence on test power, however, it can be noted that applying the parametric bootstrap to test the significance of effect estimates from boosted location and scale models satisfies the type-Ierror rate regardless of the degree of regularization.

\section{Peak expiratory flow and physical exercise}

Impaired pulmonary function is one of the main causes held responsible for disability or even death of older adults $[39,40]$. While low lung function is naturally both cause and consequence of various respiratory diseases, it has also been associated with cardio-vascular diseases, for example [41,42]. Therefore, therapies that are able to improve or maintain lung function are of great value to both patients and health care system in general. As one might probably expect, sufficient physical exercise is a suitable way to achieve higher average lung function (e.g. [43-45]). However, as overall health is dramatically worsening the lower the observed lung function, it might be of additional interest to examine the effect on the variance of the measurements, since a wider spread of the values would mean a higher proportion of patients in critical regions even without significant effect on the expectation. For this purpose, we use our suggested approaches to analyse data from the public release of the German Ageing Survey (DEAS), provided by the Research Data Center of the German Center of Gerontology (DZA) [46]. The survey comprises a sample of the German population aged 40 years and over and addresses a wide variety of topics including physical and mental health, social ressources and daily behaviour, with the latter including questions regarding the average time per week spent on sporting activities. Alongside the interview participants were also asked to perform a pulmonary function test to measure the peak flow in $1 /$ min which will be our response variable. Figure 3 shows the scatter plot for both variables. 


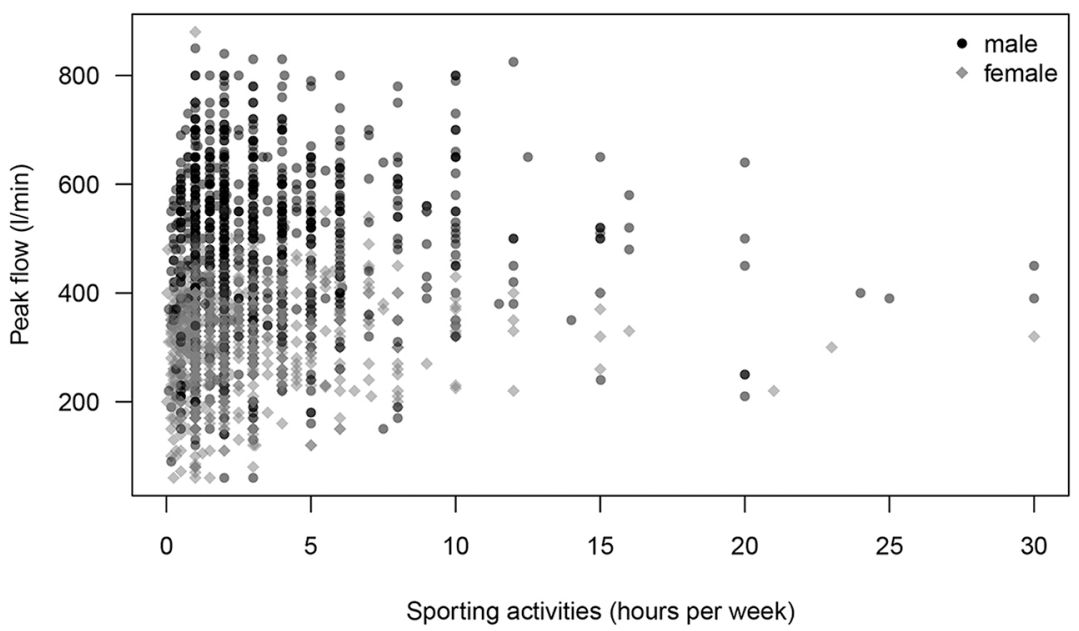

Figure 3: Pulmonary peak flow of older people in Germany and their weekly sporting activities, with black dots representing male and grey diamonds representing female study participants. Data are from the public release of the German Ageing Survey (DEAS) [46].

While controlled clinical experiments can resort to randomization, survey data such as the DEAS require potential confounders to be explicitly accounted for in the analyses in order to prevent misinterpretation of the effects one is primarily interested in. Therefore, we evaluate the effect of the amount of weekly sporting activities via the location and scale model

$$
\begin{gathered}
2 \hat{\mu} \text { (Peak flow })=\beta_{0 \mu}+\beta_{1 \mu} \text { Sports }+\beta_{2 \mu} \text { Age }+\beta_{3 \mu} \text { BMI }+\beta_{4 \mu} \mathrm{PD} \\
\log (\hat{\sigma}(\text { Peak flow }))=\beta_{0 \sigma}+\beta_{1 \sigma} \text { Sports }+\beta_{2 \sigma} \text { Age }+\beta_{3 \sigma} \text { BMI }+\beta_{4 \sigma} \mathrm{PD},
\end{gathered}
$$

using the same linear model for both distribution parameters while adjusting for age, body-mass-index (BMI) and the presence of any pulmonary disease (PD). Table 2 shows the results for both residual permutation test and the parametric bootstrap approach after fitting the model above via gradient boosting for different stopping iterations as well as the Wald-type test implemented in gamlss. While all approaches clearly agree on a significant effect of the weekly amount of sporting activities on the expected peak flow achieved in the lung function test, computing $p$-values based on boosted location and scale models leads to a higher $p$-value and, consequently, to another test decision regarding the effect on the variance when stopping the algorithm early at 50 iterations. This can be traced back to the fact that the models considered in the simulation study involved only two potentially influential variables for both distribution parameters that were hence likely to be selected early on if truly informative. As stated in the simulation section, we therefore emphasize to run the algorithm long enough for more complex data settings.

Revisiting Figure 3, however, it can be observed that female participants appear to have lower average peak flow. Moreover, gender is fairly correlated to the amount of weekly sporting activities. As a consequence, we estimated the model a second time and added a term to account for the participants gender in both $\eta_{\mu}$ and $\eta_{\sigma}$. The $p$-values resulting from this second model are shown in the lower half of Table 2. For all applied methods, the effect of weekly sporting activities is not significant any more after the additional adjustment for gender. When stopping the algorithm very early, this eventually leads to the base-learner using the sports-variable not being selected once, consequently resulting in an estimated $p$-value of 1 . While this of course does not necessarily mean that there is no true effect of the amount of sporting activities on the peak flow, the applied hypothesis tests are again in line with the results from the gamlss-package.

Table 2: p-values computed for the linear effect of weekly sporting activities on the standardized peak flow. The second model is identical to the first but additionally adjusts for the participants gender.

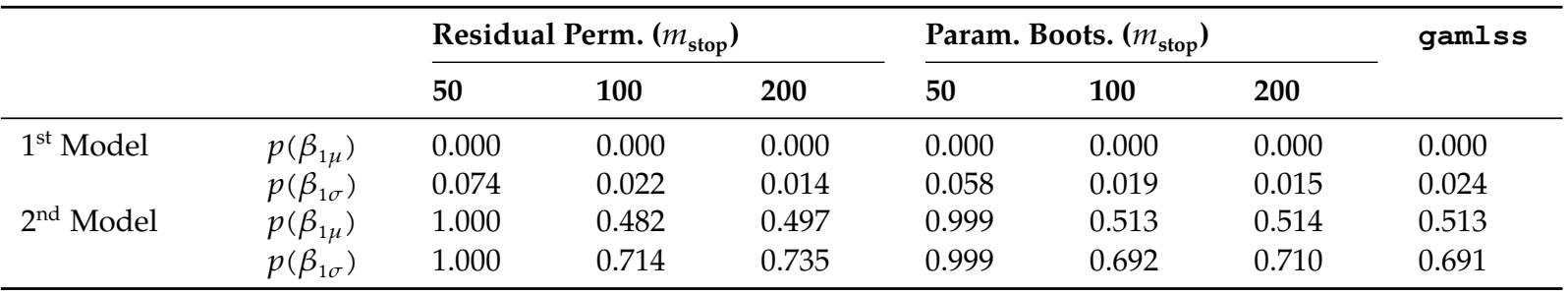




\section{Conclusion}

With penalized likelihood maximization being the gold-standard in low-dimensional generalized additive models for location, scale and shape [2], model-based boosting [4] represents a versatile alternative. By selecting only a single base-learner in each iteration of the algorithm, these type of boosting algorithms allow to fit models to high-dimensional datasets. In making use of the implicit model regularization induced by early stopping of the algorithm, boosting algorithms could further be used to perform data-driven variable selection. A drawback of the approach, however, is that the effect estimates are subject to shrinkage very similar to the lasso [27]. As a result, there exist no straight-forward way to provide similar confidence intervals or significance tests for effect estimates as in the original inference framework.

To provide some kind of error control with respect to the variable selection, earlier work focused on adapting stability selection [47] for boosting algorithms [48, 49]. However, particularly in biostatistics, researchers often not only want to name stable predictors but also want to assess the significance of single effects.

We propose two strategies that allow the calculation of $p$-values in correlated designs to further extend the scope of application scenarios for boosted location and scale models. The first, a residual permutation test, refines the basic permutation approach by permuting residuals from a linear regression instead of the original variable $[29,30]$, while the second uses the concept of parametric bootstraps to generate new response vectors under the null-hypothesis. To the best of our knowledge, this is the first time that boosting approaches are combined with either method.

Based on extensive simulations in a Gaussian location and scale framework, we were able to show that both new suggested test strategies are able to hold the type-I-error rate in the presence of correlated covariates for different amounts of shrinkage imposed on the effect estimates. Moreover, both procedures lead to reasonable test power, especially if the variable of interest is only associated to either location or scale of the response. Results for the parametric bootstrap, on the other side, show a shift of test power for small absolute values of the true effect size if the model is regularized too strongly. While the residual permutation test therefore seems to be preferable to the parametric bootstrap in terms of test power, it should be noted that its application is limited to low-dimensional scenarios due to the prerequisite for first computing the residuals via ordinary least squares in order to ensure uncorrelatedness to all other covariates.

In low-dimensional data, boosting might be advantageous towards classical inference due to variable selection and greater flexibility with respect to different type of effects [50]. For high-dimensional data with more potential variables than observations, the boosting approach is the only feasible way to fit this model class, which makes any approach to compute significance tests in those settings even more important. Results of our simulations suggest, that the power of the parametric bootstrap test decreases, but the type-I error is still under control.

We further analysed data from an epidemiological study to model the relationship between lung function and sportive activity. Leading to the same test decisions compared to each other as well as the gamlss-package used as 'gold-standard' for two different model formulations, we were able to illustrate that both suggested approaches may result in meaningful statistical tests and further insights regarding single covariates based on boosted location and scale models.

A potential drawback of the suggested approaches is that their runtime scales approximately linear in $p, B$ and the final number of boosting iterations. Considering that the test power might be affected by stopping the algorithm too early, this may require some computational efforts for more complex problems. However, as all $B$ repetitions can be run independently from each other they can be parallelized quite easily.

Since we restricted this work to linear effects in Gaussian distributed location and scale models, further research will investigate if the overall concept works with other distributional regression models as well. As the parametric bootstrap test is in fact based on the likelihood-ratio as test statistic, it should be also able to adapt it for non-linear effects. Additionally, we will investigate how our approaches perform with the recently proposed non-cyclical boosting algorithm by Thomas et al. [51]. On a broader perspective, the concept should in general also be applicable to other regularized regression techniques such as the lasso [26].

\section{Funding}

The work on this article was supported by the German Research Foundation (DFG), grant SCHM 2966/1-2 and the Interdisciplinary Center for Clinical Research (IZKF) of the Friedrich-Alexander-University ErlangenNürnberg (Project J49).

Conflict of Interest: The authors declare that they have no conflict of interest. 
Note: Tobias Hepp performed the present work in partial fulfilment of the requirements for obtaining the degree 'Dr. rer. biol. hum.' at the University of Erlangen-Nuremberg.

\section{References}

[1] Hastie T, Tibshirani R. Generalized additive models. Stat Sci. 1986;1:297-310. DOI: 10.1214/ss/1177013604.

[2] Rigby RA, Stasinopoulos DM. Generalized additive models for location, scale and shape. ] R Stat Soc: C (Applied Statistics). 2005;54:50754.

[3] Stasinopoulos DM, Rigby RA, et al. Ceneralized additive models for location scale and shape (CAMLSS) in R. ] Stat Software. 2007;23:1-46.

[4] Mayr A, Fenske N, Hofner B, Kneib T, Schmid M. Generalized additive models for location, scale and shape for high dimensional data-a flexible approach based on boosting. J R Stat Soc: C (Applied Statistics). 2012;61:403-27.

[5] Schmid M, Potapov S, Pfahlberg A, Hothorn T. Estimation and regularization techniques for regression models with multidimensional prediction functions. Stat Comput. 2010;20:139-50.

[6] Friedman ], Hastie T, Tibshirani R. Additive logistic regression: a statistical view of boosting (With discussion and a rejoinder by the authors). Ann Stat. 2000;28:337-407. DOI: 10.1214/aos/1016218223.

[7] Ridgeway G. The state of boosting. Comput Sci Stat. 1999;31:172-81. Available at: http://citeseerx.ist.psu.edu/viewdoc/summary?doi=10.1.1.22.276.

[8] Bühlmann P, Hothorn T. Boosting algorithms: regularization, prediction and model fitting. Stat Sci. 2007;22:477-505.

[9] Mayr A, Binder H, Gefeller O, Schmid M. The evolution of boosting algorithms. Meth Inf Med. 2014;53:419-27.

[10] Tutz G, Binder H. Generalized additive modeling with implicit variable selection by likelihood-based boosting. Biometrics. 2006;62:96171.

[11] Schmid M, Hothorn T. Boosting additive models using component-wise P-splines. Comput Stat Data Anal. 2008;53:298-311.

[12] Mayr A, Hofner B, Waldmann E, Hepp T, Meyer S, Gefeller O. An update on statistical boosting in biomedicine. Comput Math Methods Med. 2017; Article ID 6083072:1-12

[13] Hofner B, Kneib T, Hothorn T. A unified framework of constrained regression. Stat Comput. 2016;26:1-14.

[14] Seibold H, Bernau C, Boulesteix AL, De Bin R. On the choice and influence of the number of boosting steps for high-dimensional linear Cox-models. Comput Stat. 2017. DOI: 10.1007/s00180-017-0773-8.

[15] Meinshausen N, Bühlmann P. High-dimensional graphs and variable selection with the lasso. Ann Stat. 2006;1436-62.

[16] Leng C, Lin Y, Wahba G. A note on the lasso and related procedures in model selection. Stat Sin. 2006;16:1273-84. Available at: http://www.jstor.org/stable/24307787.

[17] Mayr A, Schmid M, Pfahlberg A, Uter W, Gefeller O. A permutation test to analyse systematic bias and random measurement errors of medical devices via boosting location and scale models. Stat Methods Med Res. 2017;26:1443-60. DOI: 10.1177/0962280215581855.

[18] Draper NR, Stoneman DM. Testing for the inclusion of variables in linear regression by a randomisation technique. Technometrics. 1966;8:695-9. Available at: http://www.jstor.org/stable/1266641.

[19] Kennedy PE, Cade BS. Randomization tests for multiple regression. Commun Stat - Simul Comput. 1996;25:923-36.

[20] Hastie T, Tibshirani R, Friedman ]. The elements of statistical learning. Springer Series in Statistics. New York, NY, USA: Springer New York Inc., 2001.

[21] Fenske N, Fahrmeir L, Hothorn T, Rzehak P, Höhle M. Boosting structured additive quantile regression for longitudinal childhood obesity data. Int ] Biostat. 2013;9:1-18.

[22] Möst L, Hothorn T. Conditional transformation models for survivor function estimation. Int] Biostat. 2015;11:23-50.

[23] Kneib T, Hothorn T, Tutz G. Variable selection and model choice in geoadditive regression models. Biometrics. 2009;65:626-34.

[24] R Core Team. R: a language and environment for statistical computing. Vienna, Austria, 2016. Available at: https://www.R-project.org/.

[25] Hofner B, Mayr A, Schmid M. gamboostLSS: an R package for model building and variable selection in the CAMLSS framework. ] Stat Software. 2016;74:1-31.

[26] Tibshirani R. Regression shrinkage and selection via the lasso. ] R Stat Soc (Series B). 1996;58:267-88.

[27] Hepp T, Schmid M, Gefeller O, Waldmann E, Mayr A. Approaches to regularized regression - a comparison between gradient boosting and the lasso. Methods Inf Med. 2016;55:422-30.

[28] Thomas ], Hepp T, Mayr A, Bischl B. Probing for sparse and fast variable selection with model-based boosting. Comput Math Methods Med. 2017;Article ID 1421409:1-8

[29] Potter DM. A permutation test for inference in logistic regression with small-and moderate-sized data sets. Stat Med. 2005;24:693-708

[30] Werft W, Benner A. glmperm: A permutation of regressor residuals test for inference in generalized linear models. R ]. 2010;2:39-43.

[31] Boulesteix AL, Hothorn T. Testing the additional predictive value of high-dimensional molecular data. BMC Bioinf. 2010;11:78.

[32] Efron B, Tibshirani R]. An introduction to the bootstrap. Boca Raton: Chapman and Hall/CRC, 1994

[33] Davison AC, Hinkley DV. Bootstrap methods and their application Vol. 1. Cambridge: Cambridge University Press, 1997

[34] Stasinopoulos MD, Rigby RA, Heller CZ, Voudouris V, De Bastiani F. Flexible regression and smoothing: using GAMLSS in R. Boca Raton: Chapman and Hall/CRC.

[35] Faraway ]]. Extending the linear model with R: generalized linear, mixed effects and nonparametric regression models Vol. 124. Boca Raton: Chapman and Hall/CRC, 2016

[36] Bühlmann P. Boosting for high-dimensional linear models. Ann Stat. 2006;34:559-83. DOI: 10.1214/009053606000000092.

[37] Li ST, Hammond ]L. Ceneration of pseudorandom numbers with specified univariate distributions and correlation coefficients. IEEE Trans Syst Man Cybern. 1975;SMC-5:557-61. 
[38] Cario MC, Nelson BL. Modeling and generating random vectors with arbitrary marginal distributions and correlation matrix. Technical Report, Department of Industrial Engineering and Management Sciences, Northwestern University, Evanston, Illinois, 1997.

[39] Sircar K, Hnizdo E, Petsonk E, Attfield M. Decline in lung function and mortality: implications for medical monitoring. Occup Environ Med. 2007;64:461-6.

[40] Roberts MH, Mapel DW. Limited lung function: impact of reduced peak expiratory flow on health status, Health-Care Utilization, and Expected Survival in Older Adults. Am J Epidemiol. 2012;176:127-34.

[41] Sin DD, Wu L, Man SP. The relationship between reduced lung function and cardiovascular mortality: a population-based study and a systematic review of the literature. CHEST ]. 2005;127:1952-9.

[42] Agarwal SK, Heiss G, Barr RG, Chang PP, Loehr LR, Chambless LE, et al. Airflow obstruction, lung function, and risk of incident heart failure: the Atherosclerosis Risk in Communities (ARIC) study. European ] Heart Failure. 2012;14:414-22. DOI: 10.1093/eurjhf/hfso16.

[43] Cheng Y, Macera CA, Addy CL, Sy F, Wieland D, Blair SN. Effects of physical activity on exercise tests and respiratory function. British ] Sports Med. 2003;37:521-8.

[44] Pelkonen M, Notkola IL, Lakka T, Tukiainen HO, Kivinen P, Nissinen A. Delaying decline in pulmonary function with physical activity: a 25-year follow-up. Am ] Respir Crit Care Med. 2003;168:494-9.

[45] Nystad W, Samuelsen S, Nafstad P, Langhammer A. Association between level of physical activity and lung function among Norwegian men and women: the HUNT study. Int ] Tuberculosis Lung Disease. 2006;10:1399-405.

[46] SUF DEAS. Version 2.0; 2011. https://www.dza.de/en/fdz/german-ageing-survey.html.

[47] Meinshausen N, Bühlmann P. Stability selection. ] R Stat Soc: B (Statistical Methodology). 2010;72:417-73.

[48] Hofner B, Boccuto L, Göker M. Controlling false discoveries in high-dimensional situations: boosting with stability selection. BMC Bioinf. 2015;16:144

[49] Mayr A, Hofner B, Schmid M. Boosting the discriminatory power of sparse survival models via optimization of the concordance index and stability selection. BMC Bioinf. 2016;17:288.

[50] Mayr A, Hofner B. Boosting for statistical modelling-A non-technical introduction. Stat Modell. 2018;18:365-84.

[51] Thomas ], Mayr A, Bischl B, Schmid M, Smith A, Hofner B. Gradient boosting for distributional regression: faster tuning and improved variable selection via noncyclical updates. Stat Comput. 2018;28:673-87.

Supplementary Material: The online version of this article offers supplementary material (DOI:https://doi.org/10.1515/ijb-2018-0110/suppl/ijb-2018-0110ad.zip). 